\title{
Computation of electronic proprieties of the DNA and RNA bases
}

\author{
Mariana Virginia Popa \\ Electronic and Telecomunication, Autonomous University of the Hidalgo State, Mexico
}

Email address:

virginia_popa@yahoo.com.mx

\section{To cite this article:}

Mariana Virginia Popa. Computation of Electronic Proprieties of the DNA and RNA Bases. International Journal of Computational and Theoretical Chemistry. Vol. 2, No. 4, 2014, pp. 26-40. doi: 10.11648/j.ijctc.20140204.11

\begin{abstract}
In this paper the authors report the optimizations of the DNA and RNA bases (adenine, cytosine, thymine, guanine and uracil) for to determine the electronic proprieties and are employed the LSDA/6-31++G, PBE/6-31++G, $\mathrm{PBE} / \mathrm{LANL} 2 \mathrm{DZ}$ and PBE/SDD levels of theory both in gas phase and in the presence of the solvent water with the actual implementation of the polarized continuum model of Tomasi (PCM). And to provide the IPV, EAV, hardness, dipole moment and electronegativity $(\chi)$. The vibrational frequencies are description to purine and pyrimidine bases.
\end{abstract}

Keywords: Purine and Pyrimidine, Electronic Proprieties, DFT for DNA and RNA

\section{Introduction}

The importance end preoccupation in the field of the biochemistry and theoretical chemical-physics is to know the active centre in the ADN and RNA chain. On the other hand, the interaction of the metals with the ADN and RNA bases ${ }^{1-9}$ has been the subject of numerous publications. The ab initio calculations as the properties such the electron affinities (EAs) and ionization potentials (IPs) have been presented en meany works with different levels of theory and are reported experimental data also ${ }^{10-25}$. The difference between vertical IPs, vertical EAs and their adiabatic counterparts consist by the nuclear reorganization energy on ion formation. The positive values for the electron affinities show the exothermic process and thermodynamically stable state ${ }^{16-22}$. It is now well established that the pyrimidine are better electron acceptors than the purine. In another publication a comprehensive and reliable computational study of the first ionization energies of the DNA and RNA bases have been calculated in the gas fase and in aqueous solution at HF and MP2 levels of theory and $6-31++G(d, p)$ base; upon where HF level in gas phase underestimated the ionization energies by ca. $1 \mathrm{eV}$ and for guanine the theoretical data is in good agreement with the experimental value in aqueous solution ${ }^{17}$. All of the calculations in order to determined vertical IPs presented by David ${ }^{23}$ for the canonical DNA bases are based on optimization with Moller Plessetperturbational theory (MP2), or the B3LYP functional, in conjunction with a different basis sets with Polarized Continuum Model in water and gas phase. The results are very close to the experimental data with exception when MP2 theory are used, the dates of vertical ionization potentials are overestimate. Norinder ${ }^{24}$ are investigate the geometries for different tautomers of the nucleic bases using the AM1 method bat in the present work is not of interest because all structure are en stable form. Comparison of calculated and experimental value the bond length and ionization potentials are overestimated in these systems. As the DNA bases is very popular benchmark molecules the papers ${ }^{[25-27]}$ mentioned the bond length and angles, vibrational frequencies. $\operatorname{In}^{28}$ the plane wave basis set in conjunction with ultrasoft pseudopotentials yield bond lengths and angles are close to conventional quantum chemistry results. In this regard all geometries of DNA bases were performed using PW91/6-311G(2df,2pd) and MP2 theory (cc-pVTZ, aug-cc-pVTZ and cc-pVQZ larger basis) used PQS Parallel Quantum Solutions, the bond length deviations are possible for the plane-wave/ultrasoft pseudopotentiales method. When predict the bond length and angle for large systems it is useful to introduce PW91. For the best prediction of the reactivity to the AND and RNA bases is very important view the shape of the SOMO follows the spin density distribution of the anion radical in their neutral geometries ${ }^{29}$. In the literature has written many papers where mention the influence of the charge, the HOMO and LUMO energies, the hardness, Fukui Functions condensate, and electronegativity for the better understand of the reactivity of the molecule in gas phase and different solvents. Since there are difficulties in determining the experimental 
ionization potentials (IPs), adiabatic electron affinities of ADN and RNA bases and the active centers to, the goal of the present work is to perform the results employed ionization potentials and electron affinities vertical, and electronegativity, for describe the possible mechanism of reaction the adenine, cytosine, guanine and uracile with different chemical reagent. So regard than Mulliken charge are not a very good describer of the activity of the atoms in the molecule for this reason in this paper we employed the NPA and CHELPG charge to.

\section{Methods}

All of the calculations presented here are optimized in gas phase and aqueous medium with $\epsilon=78.39$ (are employed Thomasi PCM model ${ }^{30}$ ). In the present work are employed DFT method with PBE and LSDA functional in combination with several 6-31++G, SDD, LANL2DZ basis $\operatorname{set}^{31}$. The full optimizations of the DNA/RNA single bases guanine $(G)$, adenine $(\mathrm{A})$, cytosine $(\mathrm{C})$, and uracil $(\mathrm{U})$ structures are also performed with Gaussian $03 \mathrm{~W}$ program package ${ }^{32}$. The optimizations were followed by frequency calculations at the same level and for the HOMO and spin density are visualized with Gauss View computational program.By definition, the vertical EA (VEA) is the energy released when an electron is added to a neutral molecule and calculated as an energy difference between the anion and the neutral molecule evaluated at the geometry for the neutral parent species. The energy of vertical ionization potentials (VIP) are calculated as an energy difference between the cationic and the neutral molecule evaluated at the geometry for the neutral DNA and RNA bases.

The absolute hardness is the resistance of the chemical potential to change in the number of electrons ${ }^{33}$.

The electronegativity $(\chi)$ of an atom or molecule is the negative of the chemical potential $\mu$ of its electronic cloud:

$$
\chi=-\mu=(\partial E / \partial N)_{V}
$$

Operational definitions of $\chi$ and chemical hardness $(\eta)$ are provided by the finite difference approximation $\eta=(I-A) / 2, \chi=1 / 2(I+A)$, where $I$ and $A$ are the ionization potential and electron affinity of the species in question ${ }^{34}$.

\section{Results and Discussion}
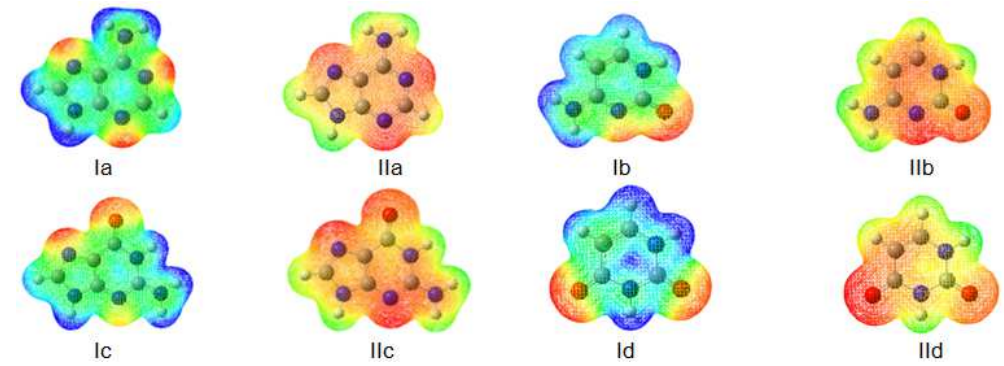

IId

Fig 1. The surfaces of the electron density mapped with ESP (isovalue $=0.006)$ of the DNA and RNA bases optimized with all level of theory employed in this work in gas phase and solvent water also $(\varepsilon=78.39)$ for neutral (I) and anion structure (II): a) adenine, b) cytosine, c) guanine and d) uracile.

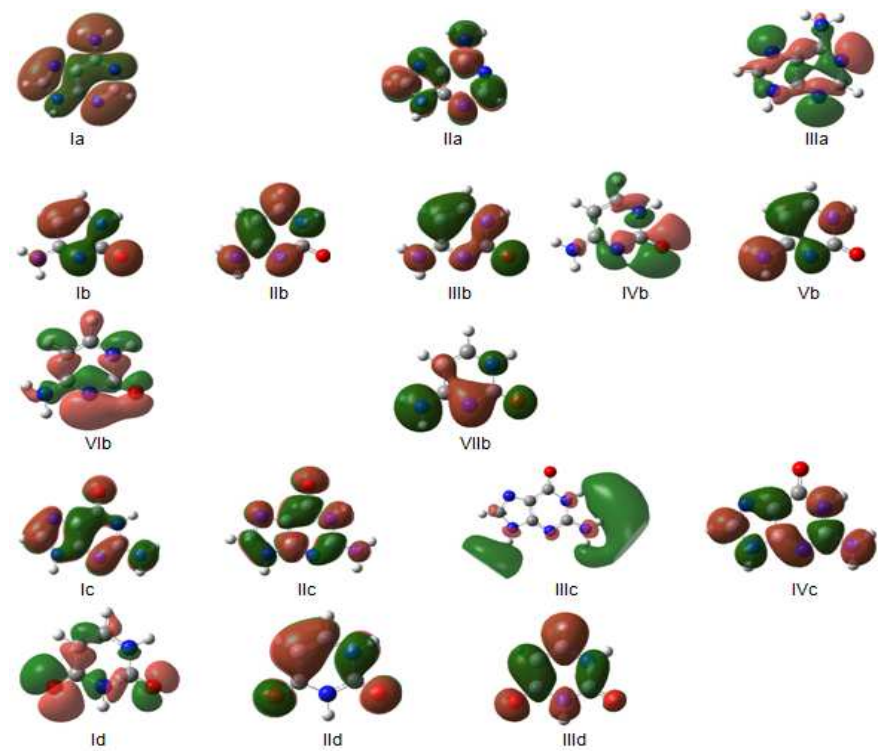

Fig 2. The HOMO and LUMO (isovalue = 0.02) of the DNA and RNA bases optimized with all level of theory employed in this work in gas phase and solvent water also ( $\varepsilon=78.39)$ for neutral HOMO and LUMO, anion HOMO and cation HOMObases; a) adenine, b) cytosine c) guanine and d) uracile. 


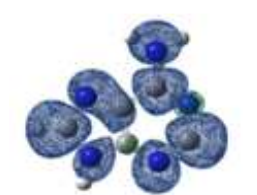

la

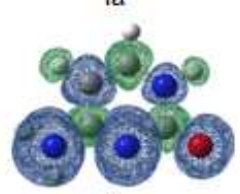

$\mathrm{Ib}$

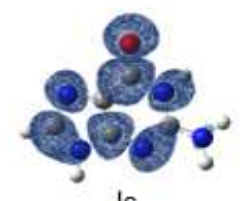

Ic

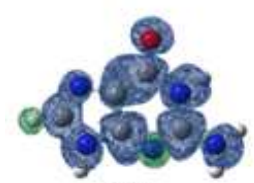

Vc

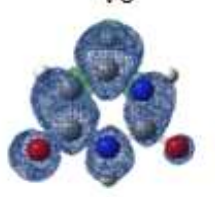

Id

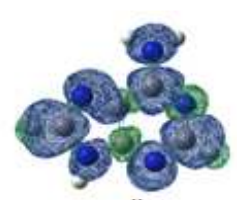

lla

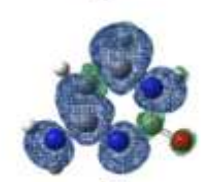

$\mathrm{Ilb}$

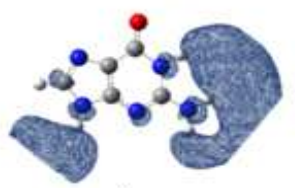

Ilc

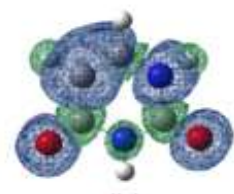

IId

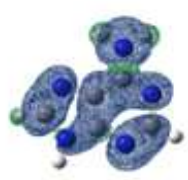

IIla

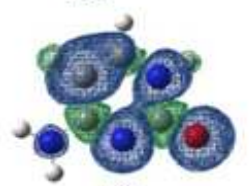

IIIb

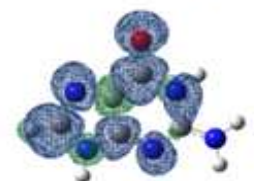

IIIC

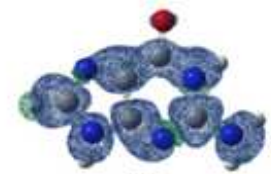

IVC

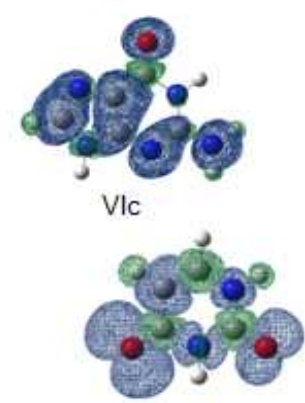

IIId

Fig 3. Spin density of anion and cation on the geometry of the optimized neutral structure (isovalue =0.004) (IV): a) adenine, b) cytosine, c) guanine, and d) uracile.
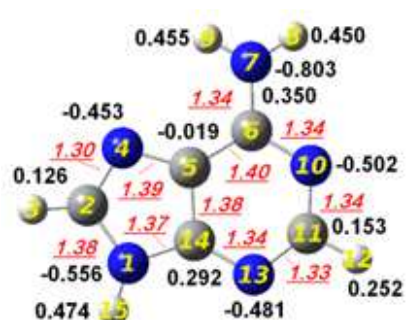

la
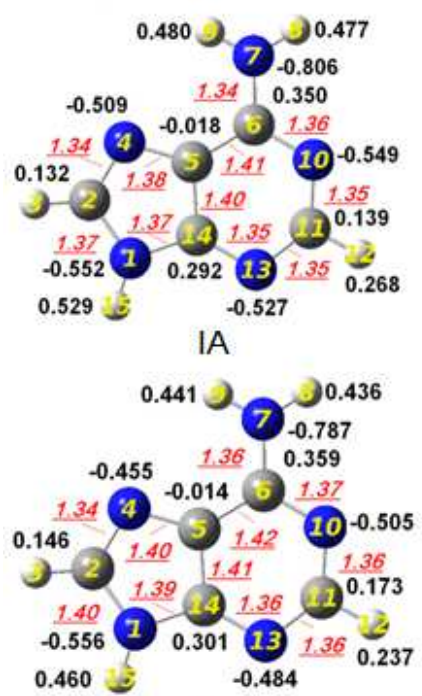

Ila

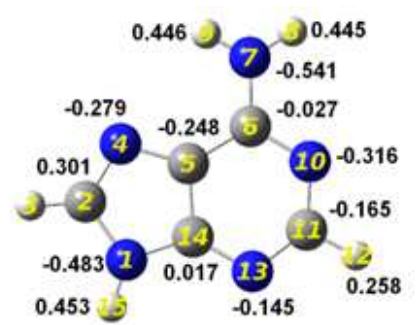

0.453 - 0.145
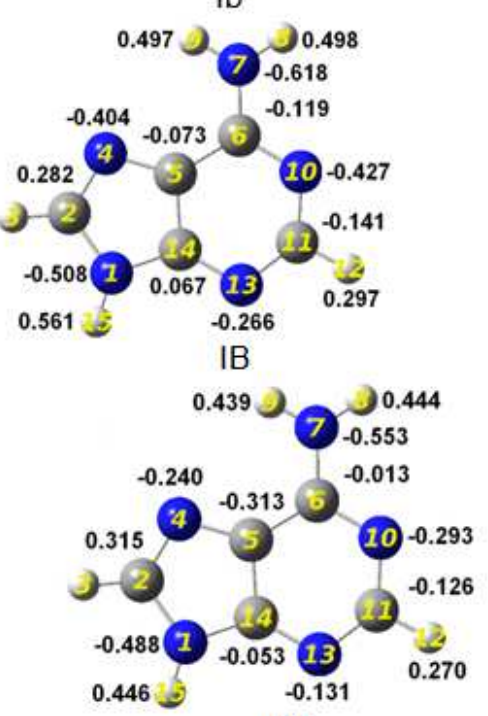

IIb
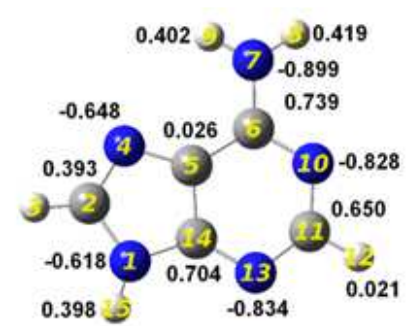

Ic
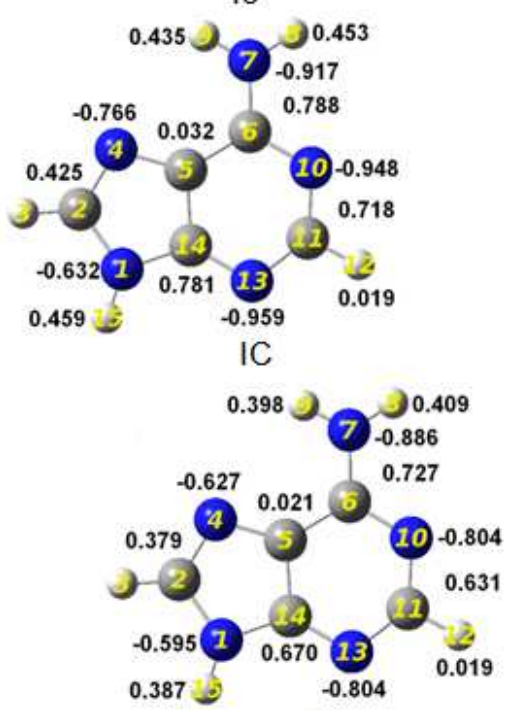

IIC 

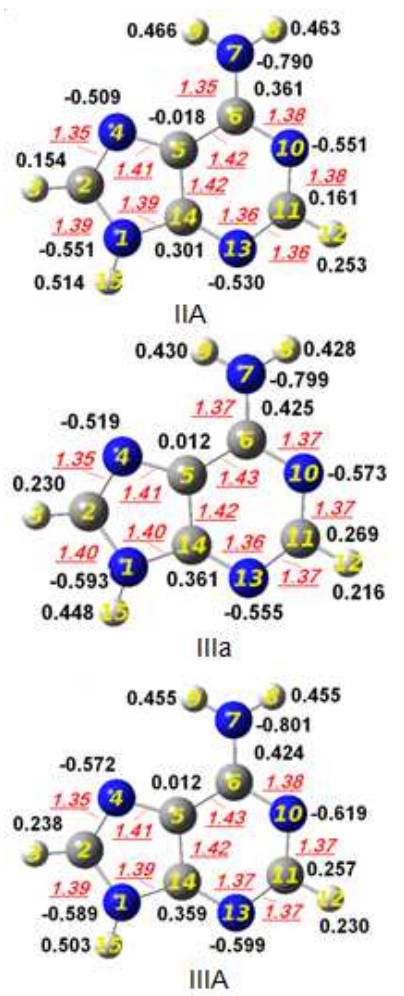
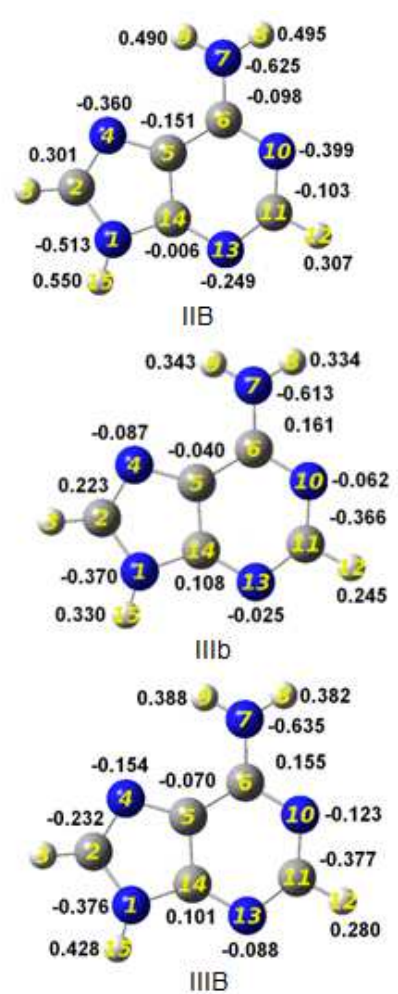
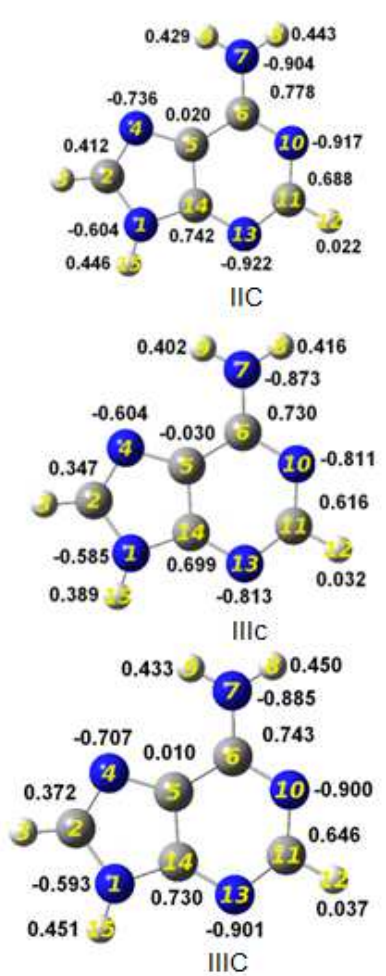

Fig 4. The charges of adenine. Bond lengths in A.I - optimized with LSDA/6-31++G level of theory, II - optimized with PBE/6-31++G level of theory, IIIoptimized with PBE/LANL2DZ or PBE/SDD level of theory; a-NPA, b-Mulliken, $c$ - CHELPG charge in gas phase; $A$ - NPA, B - Mulliken and C - CHELPG charge in solvent water $(\varepsilon=78.39)$.

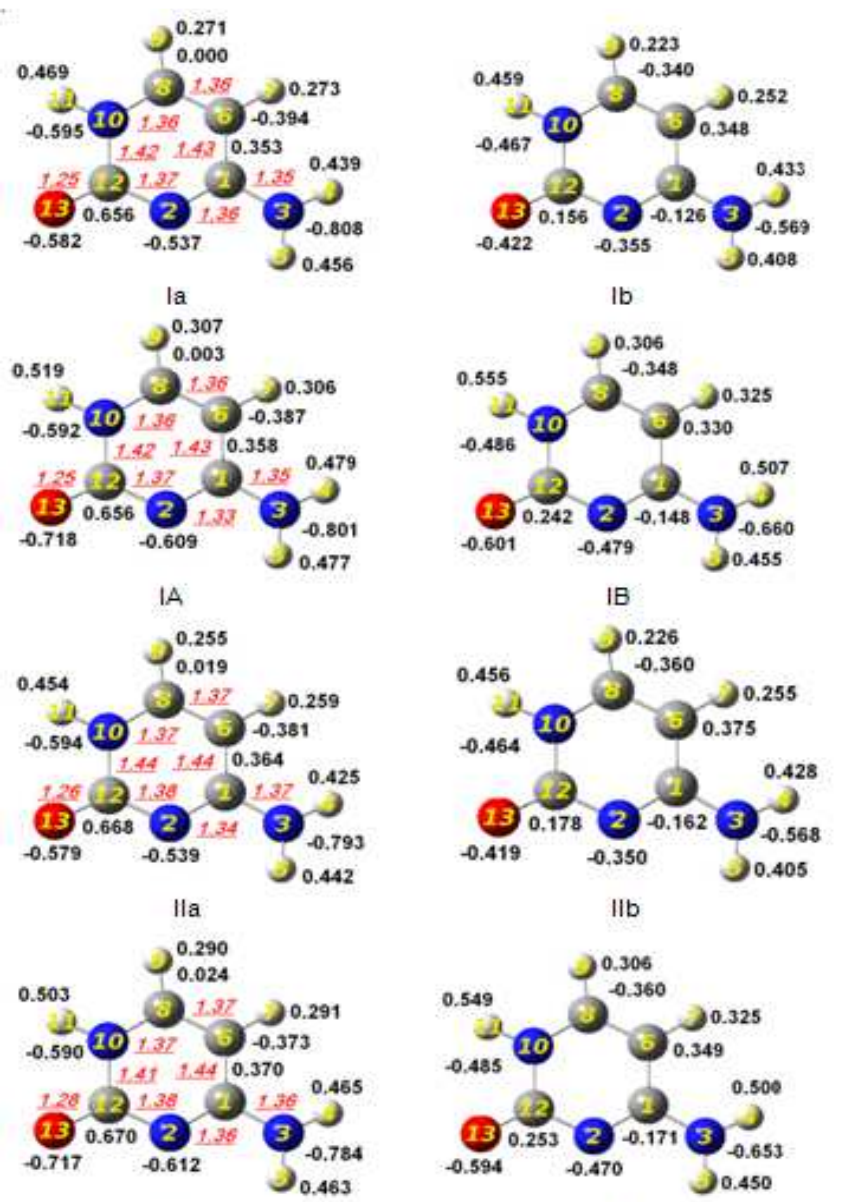

IIA

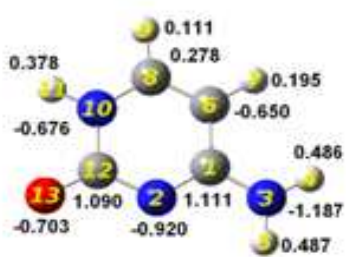

Ic

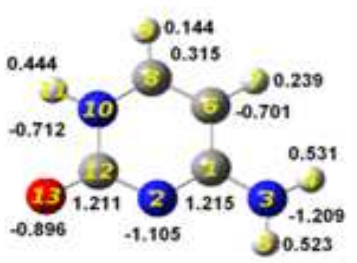

IC
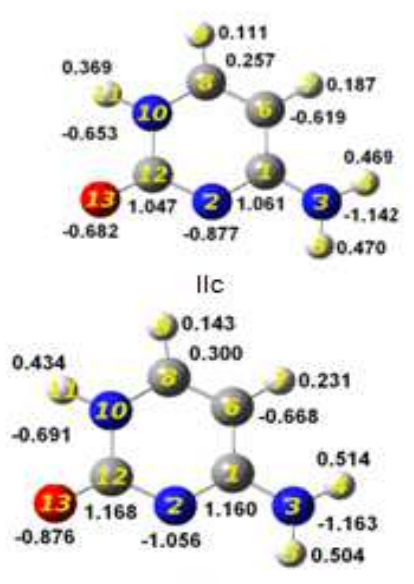

$I I C$ 

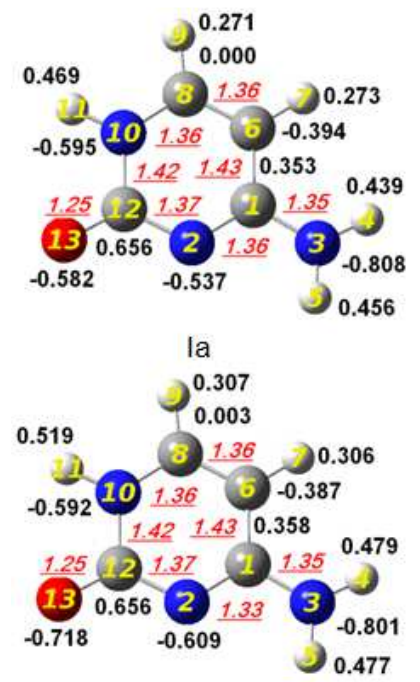

IA

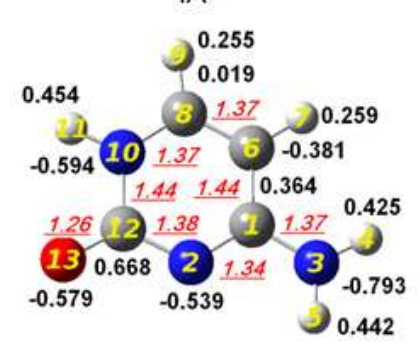

IIa

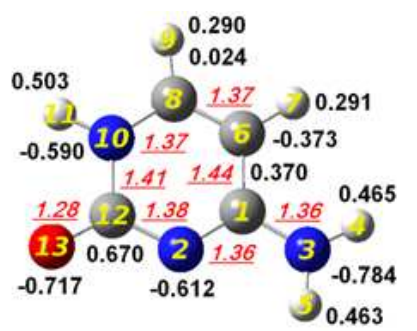

IIA

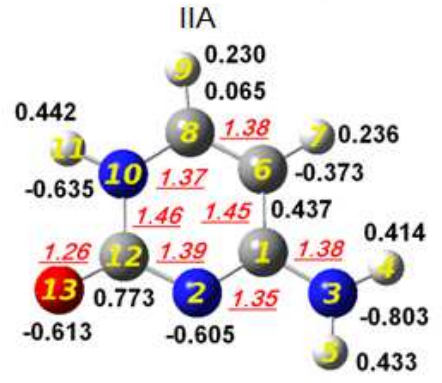

IIla

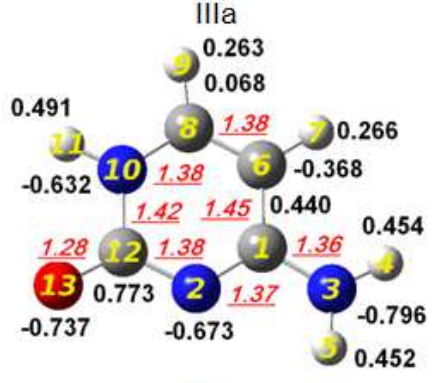

IIIA

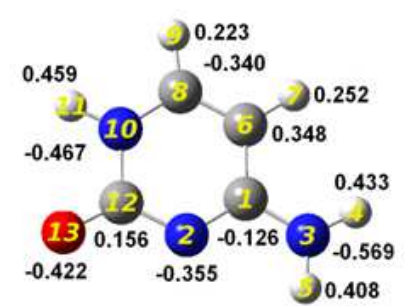

Ib

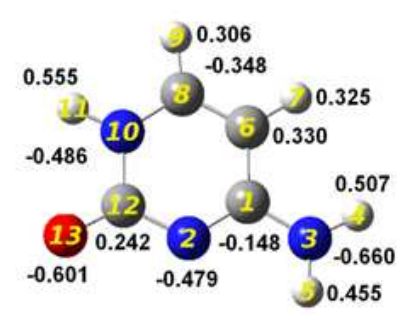

IB

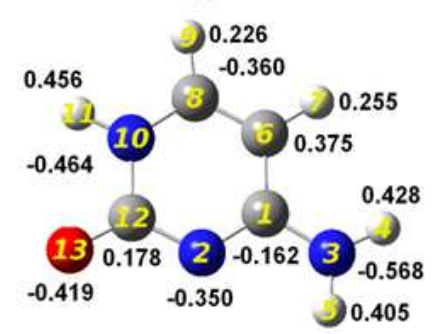

IIb

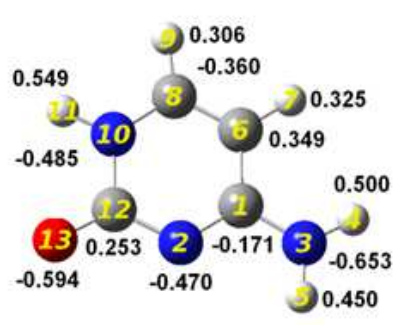

IIB

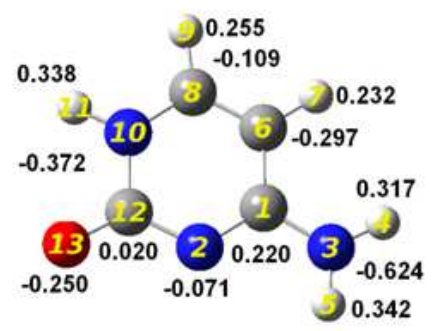

IIIb

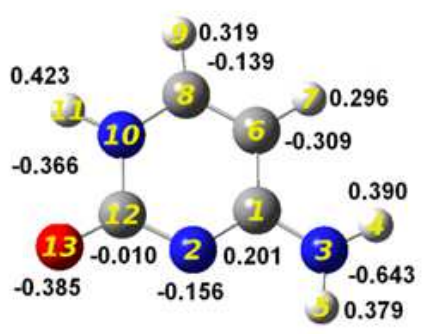

IIIB

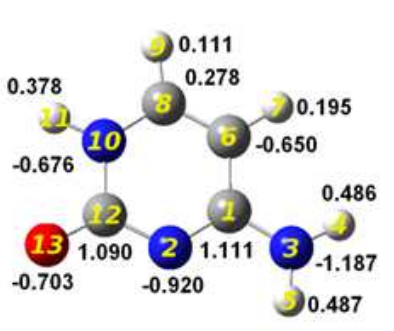

Ic

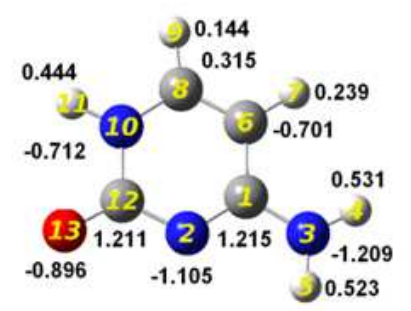

IC

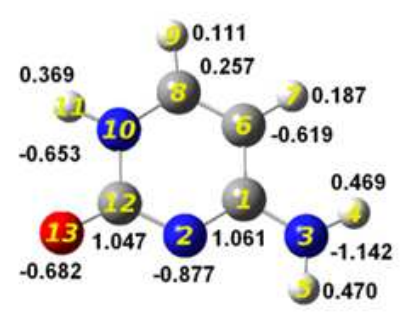

IIC

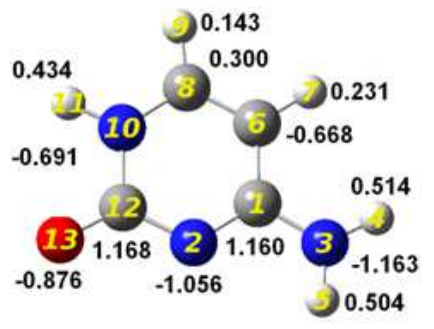

IIC

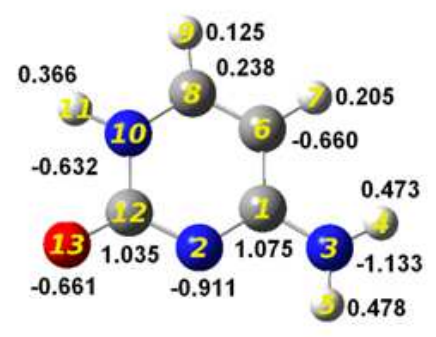

IIIC

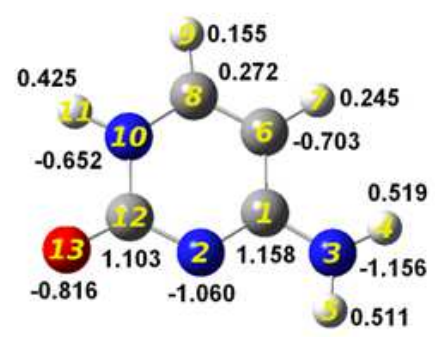

IIIC

Fig 5. The charges of cytosine. Bond lengths in A. I - optimized with LSDA/6-31++G level of theory, II - optimized with PBE/6-31++G level of theory, III optimized with PBE/LANL2DZ or PBE/SDD level of theory; a-NPA, $b$-Mulliken, $c$ - CHELPG charge in gas phase; $A$ - NPA, $B$ - Mulliken and C - CHELPG charge in solvent water $(\varepsilon=78.39)$. 


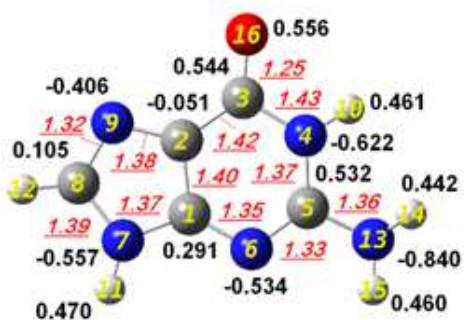

la

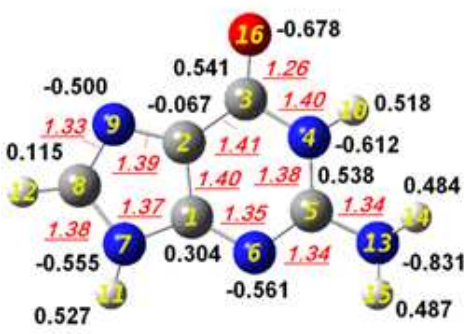

IA

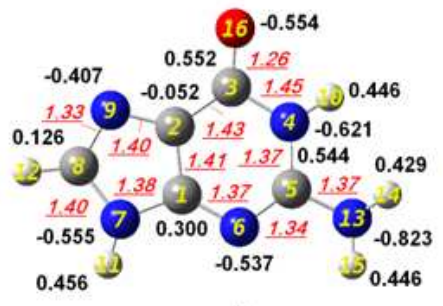

Ila

(16) -0.697

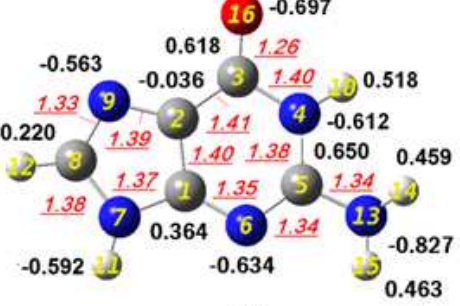

IIA

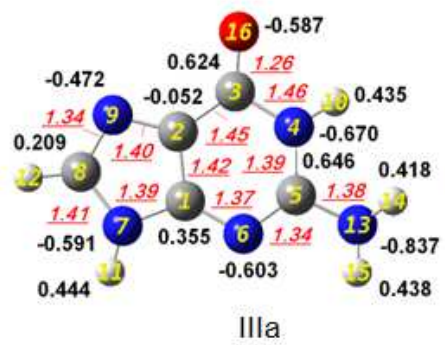

IIla

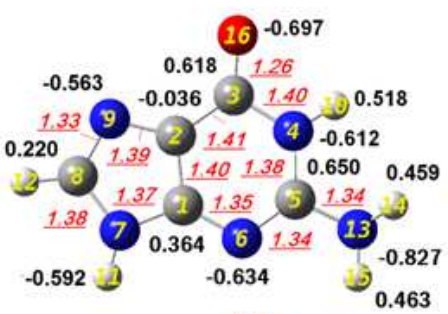

IIIA

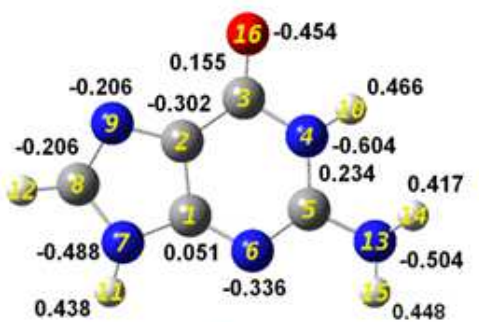

$\mathrm{Ib}$

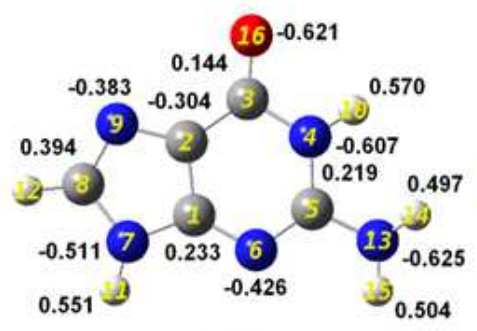

IB

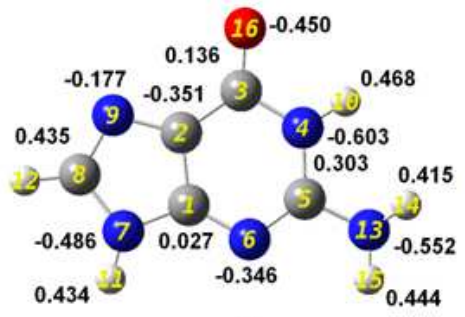

$\mathrm{Ilb}$

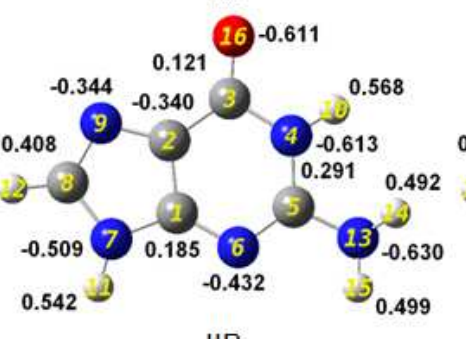

IIB

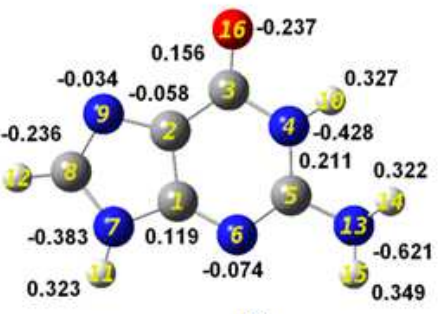

IIIb

(16) -0.362
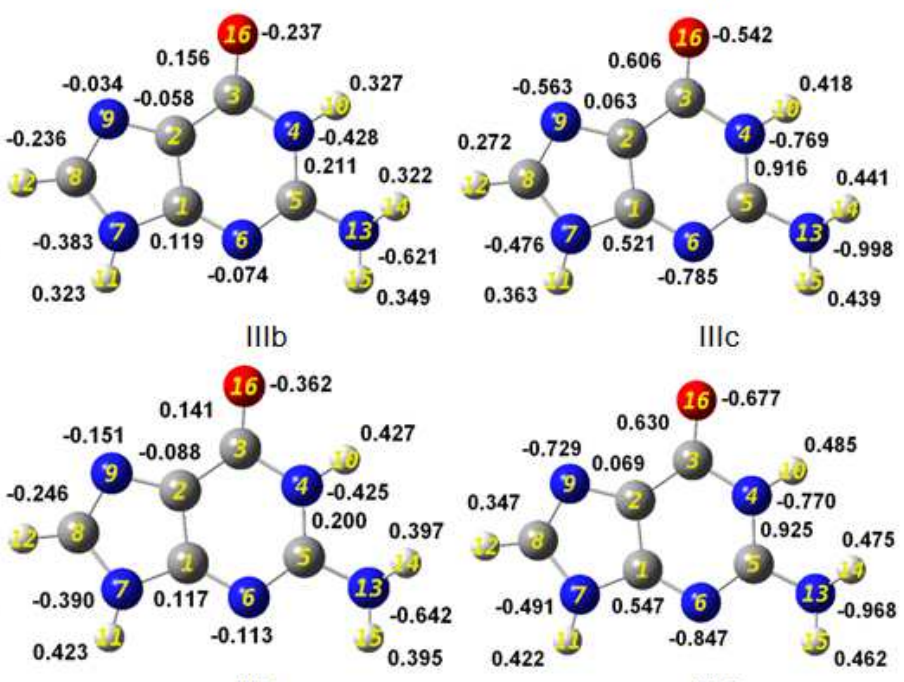

IIIC

IIC

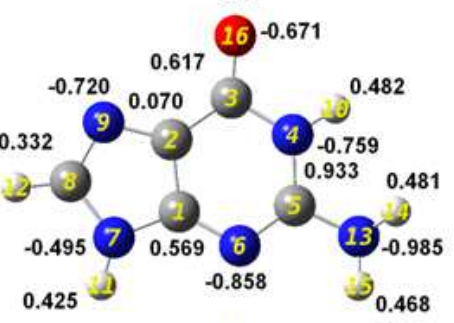

IIC 

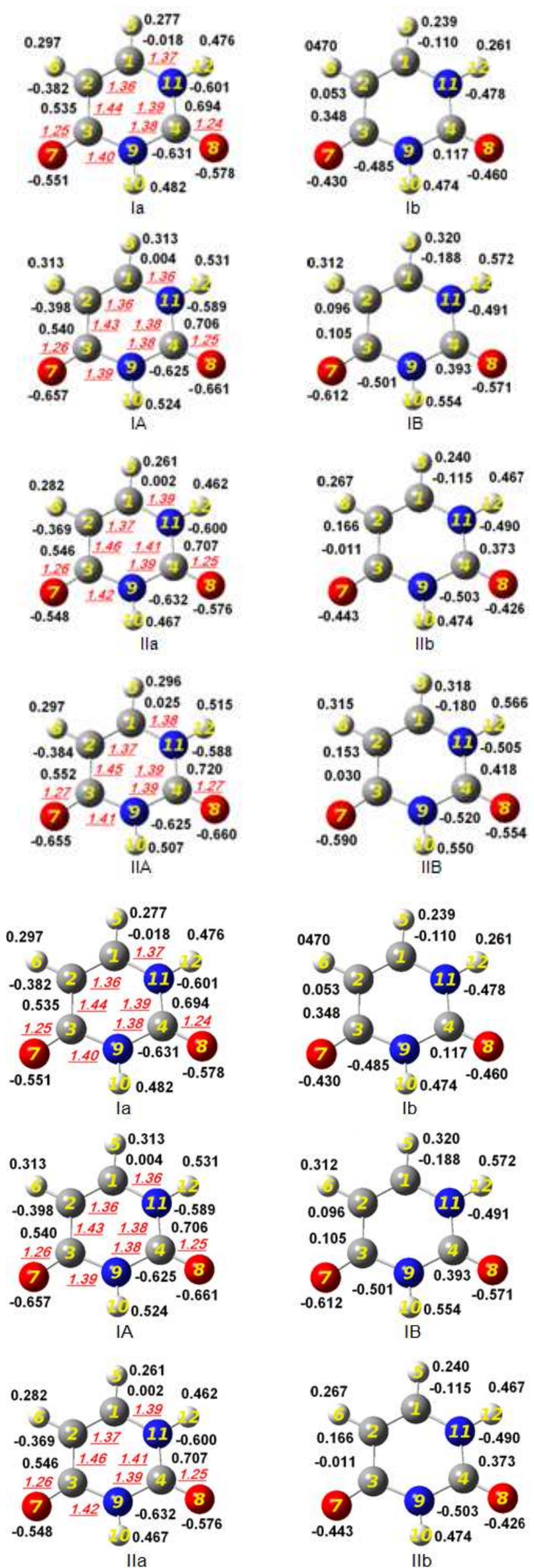
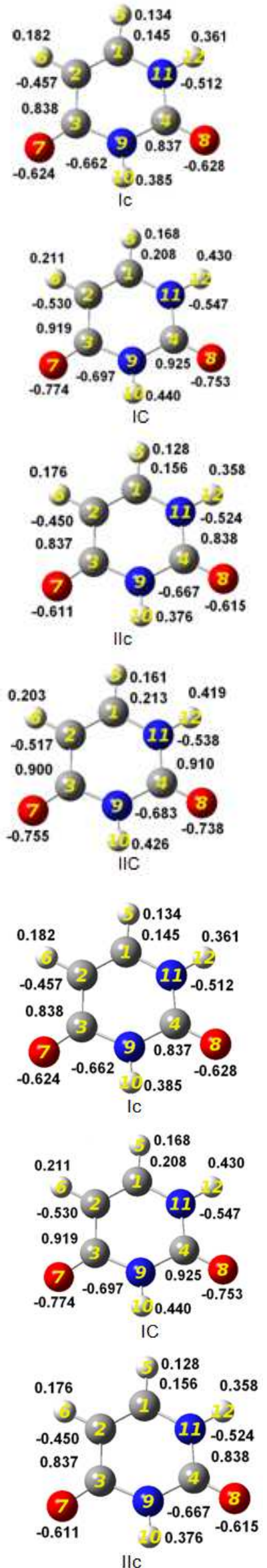

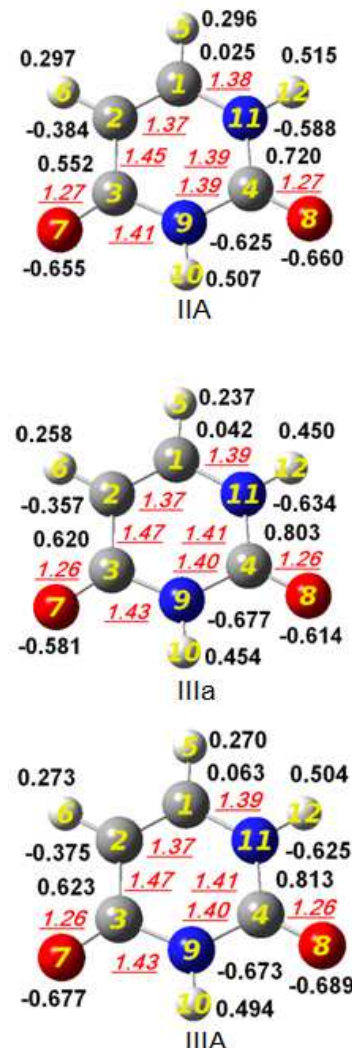
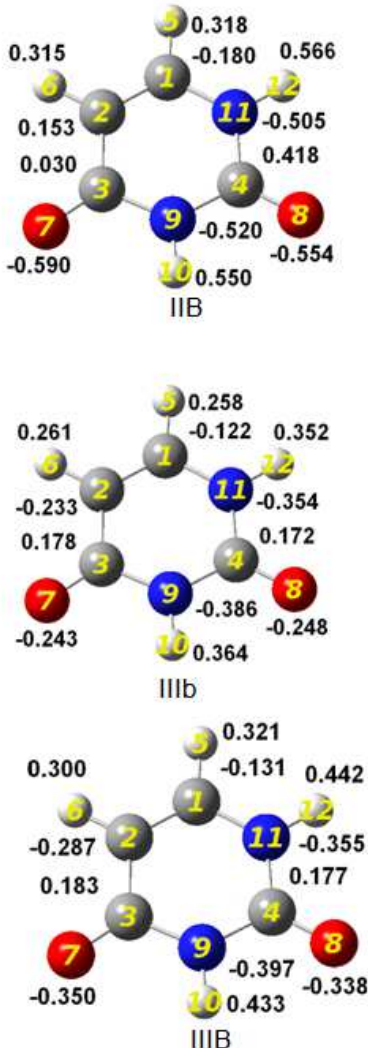
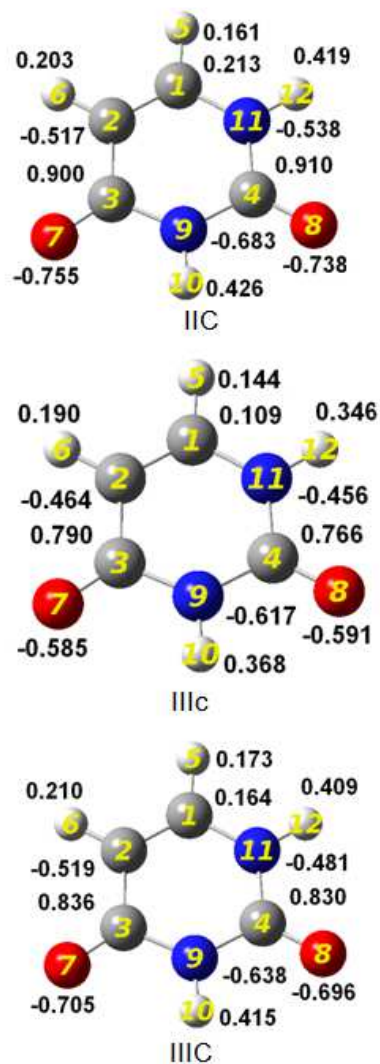

Fig 7. The charge of uracile. Bond lengths in A. I - optimized with LSDA/6-31++G level of theory, II - optimized with PBE/6-31++G level of theory, III optimized with PBE/LANL2DZ or PBE/SDD level of theory; - NPA, $b$ - Mulliken, $c$ - CHELPG charge in gas phase; $A$ - NPA, B - Mulliken and C - CHELPG charge in solvent water $(\varepsilon=78.39)$.

The aim of this work was therefore to reinvestigate the relative stabilities of the cytosine, thymine, adenine, guanine and uracile using DFT method with full geometry optimization. This procedure has proved to be useful in the prediction of such molecular properties as the geometries, dipole moments, hardness, electronegativity, electron affinity, harmonic frequencies, the surfaces of the electron density mapped with ESP and ionization potentials, the HOMO and LUMO, spin density of the anion in the neutral structure. Although chemical reactivity is characterized by global reactivity parameters like electronegativity or hardness, the selectivity is usually understood in terms of local functions like the Fukui function $\mathrm{f}(\mathrm{r})$ and local softness $\mathrm{s}(\mathrm{r})$. These global and local descriptors of reactivity have been popularized within the framework of conceptual density functional theory, a field to which reviews have been dedicated recently.

The charge transfer in DNA and RNA is the fundamental interest of the reactivity by attaching or intercalating donor and acceptor chromophores electronically coupled via the bridge orbital. The interaction between electrophiles (agents with deficit of electrons) and the AND and RNA bases is a complex phenomenon. In the previous experimental investigations ${ }^{35,36}$ the products of the interaction of the three methyllating agents characterized by increasing carcinogenic activity with ADN and ARN bases have also been examined by means of Raman and IR spectra. In this regard in the Fig. 1 I show the reactivity of adenine, cytosine, guanine, thymine and uracile for neutral and anion structure where the red part represent the electrophylic center, the green part represent the centre of posible radical attack and the blue drawing the atoms susceptibles to nucleophylic attack. The charge is disposed above all atoms in the molecules when an electron is accepted because is possible the aromaticity to disappear, but the electrophylic centersare the same excepting for the guanine when N6 is new for the $\pi$ length, Fig. 1 (Ic and IIc), Fig. 2 (IIc and IVc). N7, N10 and N4 are the most probable electrophylic center thus as has mentioned previously ${ }^{37}$ employed $\mathrm{PBE} / 6-31++\mathrm{G}$ and LSDA/6-31++G levels of theories, and in this work Fig. 1 (Ia) for adenine. Is very know which the surfaces of the electron density mapped with ESP are not very good describer of the active centers but for larger molecules are a good method because employed Fukui Functions for the optimized structure of DNA and RNA with DFT is expensive in time.

The Fig. 2 is very useful for predict the $\pi$-conjugated molecular and delocalized frontier orbitals in the negatively molecule charged, for this reason the HOMO and LUMO can be used as indicators able to predict electrical characteristics in a first approximation of single molecules. For adenine en Fig. 2 (Ia and IIa) the HOMO and LUMOen gas phase and solvent water not change with the inclusion of the relativistic proprieties and the difference between PBE and LSDA. Remove one electron of the adenine in the HOMO denote the cation change. The cation structure of cytosine in the Fig. 2 ( Vb, VIb y VIIb) are very different of the neutral and anion 
structure. On the other hand, for guanine in the HOMO cation and neutral configuration Fig. 2 (Ic) are in discrepancy with the another (Figure 2 IIc) where the antibonding exist. The diffuse bases set is the possible accountable for the great contrariety regarded in the Fig. 2 (IIc and IVc). The solvent water (l) stabilize the uracile Fig. 2 (IId, IIId) HOMO and LUMO with the HOMO cation PBE/6-31++G and PBE/SDD levels of theory exception Fig. 2 (Id). The spin surfaces of the cation Fig. 3 (IIIa) with PBE and all neutral Fig. 2 (Ia) is very similar, however with $\mathrm{LSDA} / 6-31++\mathrm{G}$ cation, $\mathrm{PBE} / \mathrm{LANL} 2 \mathrm{DZ}$ cation and $\mathrm{PBE} / \mathrm{SDD}$ cation in gas phase Fig. 3(IVa) and HOMO LSDA/6-31++G cation in gas phase and all cation in gas phase and solvent water Fig. 2 (IIIa) are identical. For adenine the results obtained in gas phase and solvent was not found to be very important in the description of the chemical proprieties. In the literature ${ }^{29}$ has been reported the SOMO surface and spin density of the anion radical of the purine and pyrimidine bases in the gas phase and including the effect of solvating (PCM model). In cytosine, Fig. 2 (Ib, IIIb) with Figure 3 (IIIb) are antibonding orbital but very different to SOMO surfaces and spin density of the cytosine optimized with B3LYP/D95V+D and B3LYP/6-311++G(2dp) basis sets ${ }^{29}$. The HOMO LSDA/6$31++\mathrm{G}$ in gas $(\mathrm{g})$ phase Fig. $2(\mathrm{Ib})$ when least expected is not different of the Fig. 2 (IIIb) for the local spin density approximation in the water and $\mathrm{PBE} / 6-31++\mathrm{G}(\mathrm{g}, 1)$, PBE/LANL2DZ(1) and PBE/SDD(l). In Fig. 2 (Vb, VIIb) HOMO PBE/6-31++G cation, HOMO PBE/LANL2DZ cation, HOMO PBE/SDD cation in gas phase and solvent water are identical but HOMO LSDA/6-31++G cation is different, Fig. 2 (VI, VII) for the local spin density (LSD) approximation.

In an unrestricted wave function, it is convenient to define a spin density $\rho^{S}(r)$ by $^{30}$

$$
\rho^{S}(r)=\rho^{\alpha}(r)-\rho^{\beta}(r)
$$

where electrons of $\alpha$ and $\beta$ spin have different spatial distributions $\left(\rho^{\alpha} \neq \rho^{\beta}\right)$.

It is clear that in regions of space where there is a higher probability of finding an electron of $\alpha$ spin then there is of finding an electron of $\beta$ spin the spin density is positive. Alternatively, the spin density is negative in regions of space where electrons of $\beta$ spin are most prevalent.

For adenine, Fig. 3 (Ia), the spin density is positive for the electron of $\alpha$ spin, when LSDA/6-31++G(g) and PBE/6$31++\mathrm{G}(\mathrm{g})$ are used. Employed LSDA/6-31++G(1) for cation and anion, PBE/6-31++G (1) anion, PBE/LANL2DZ (g,l) for anion and $\operatorname{PBE} / \operatorname{SDD}(g, 1)$ for anion also in Figure 3 (IIa) the electron of $\beta$ spin are un little larger the spin density that adenine structure in the Fig. 3 (Ia); with of the inclusion of diffuse functions in basis sets performed of B3LYP/D95+(D) level $^{29}$ adenine are the same reactivity centers.

In Fig. $3(\mathrm{Ib})$ where are employed LSDA/6-31++G cation in water solvent $\mathrm{N} 2 \mathrm{y} \mathrm{N} 3$ are when least expected the $\beta$ spin greater that another atoms. The structure of the anion in Fig. 3 (IIb) is very different of the cation structure.

Fig. 2 (Ic) show the structure of the HOMO LSDA/6-
$31++\mathrm{G}(\mathrm{g}, 1)$, HOMO LSDA/6-31++G cation (g,l), HOMO $\mathrm{PBE} / 6-31++\mathrm{G}(\mathrm{g}, 1), \quad \mathrm{HOMO} \quad \mathrm{PBE} / 6-31++\mathrm{G}$ cation $(\mathrm{g}, 1)$, HOMO PBE/LANL2DZ(g,l), HOMO PBE/LANL2DZ cation(g,l), HOMO PBE/SDD(g,l), HOMO PBE/SDD cation $(\mathrm{g}, \mathrm{l})$ when is very conspicuous the aromaticity and the $\pi$ length N9-C8, N6-C5. With an electron more in anion structure to regard the anti- $\pi$ length. LUMO LSDA/6$31++\mathrm{G}(1)$, HOMO LSDA/6-31++G anion(1), LUMO PBE/6$31++\mathrm{G}(1)$, HOMO PBE/6-31++G anion(l), LUMO PBE/6$31++\mathrm{G}$ anion(1), LUMO PBE/LANL2DZ(g,1), LUMO PBE/SDD(1), HOMO PBE/SDD anion(l), HOMO PBE/LANL2DZ anion(l) in the Fig. 2(IIc)are $3 \pi$ lenght with $3 \mathrm{p}$ orbitals alternatively disposed. The spin density of anion with the optimized structure at LSDA/6-31G(g) level of theory are similar with Fig. 2 (IIc). In HOMO PBE/LANL2DZ anion(g) Figure 2 (IVc) the $\pi$ length are different disposed in comparison with the Figure2 (IIc). In the Figure 3 (IIc) where guanine is optimized with LSDA/6$31++\mathrm{G}(\mathrm{g})$ are similar arrangement of the electron with HOMO anion in Figure 2 (IIIc), it is clear that in this regions of space is a higher probability of finding an electron of $\alpha$ spin and for this reason the spin density is positive. In the regions of the $\mathrm{C} 2$ and $\mathrm{N} 7$ are electron of $\beta$ spin when $\mathrm{PBE} / 6$ $31++\mathrm{G}$ (1), PBE/LANL2DZ (1), LSDA/6-31++G (1), PBE/SDD (l) anion (g) are used, Fig. 3 (IIIc). In Fig. 3 (IVc) the anion $(\mathrm{g})$ when I performed with PBE/LANL2DZ level the contour of anion (g) HOMO, Figure 2 (IVc), is similar with the spin density, in the regions of space when are $\alpha$ spin is positive. In Fig. 3 (IVc)and (Vc) when I employed PBE/LANL2DZ and PBE/SDDlevels of theory, in gas phase, for anion structure, the guanine are very similar with the HOMO in the Fig. 2 (IVc) and (IIc), the difference of the LANL2DZ and SDD are picture in the $\pi$ length. The LANL2DZ relativist basis in Fig. 3 (IVb) are not very different in the active centers with PM3, D95V(D), 6-31G(D) and $6-31+\mathrm{G}(\mathrm{D})^{29}$. The $6-311++\mathrm{G}(2 \mathrm{dp})$ and $6-31++\mathrm{G}(\mathrm{D})$ reported in literature ${ }^{9}$ and Fig. 3 (IIc) with diffuse functions suggest the shapes around of the N4, C5, N13, N6 and N7 atoms where the reactivity are better.

In Fig. 3 (VIc) for cation with $\operatorname{PBE}(\mathrm{g}, 1)$ and all bases are not difference with spin density is positive, but with LSDA $/ 6-31++\mathrm{G}(\mathrm{g}, 1)$ the distribution of the electron is the another form in the Fig. 2 (Ia).

For uracile in Figure 3 (Id) the spin density anion for all levels of theory are the exception with another purine and pyrimidine and is very different with the shape obtained employed D95+V(D). Figure 2 (Id) uracile are optimized with $\mathrm{LSDA} / 6-31++\mathrm{G}(\mathrm{g})$ for HOMO and in the water and gas phase for cation, for $\mathrm{PBE} / 6-31++\mathrm{G}$ and $\mathrm{PBE} / \mathrm{SDD}$ are the same situation when the orbital of the $\mathrm{O}$ are better reactivity; LANL2DZ in water with PBE are not the same contour. The reactivity are specifically for cation with LSDA/6-31++G(1) and $\mathrm{PBE} / 6-31++\mathrm{G}(\mathrm{g})$, in $\mathrm{O}$ atoms, is describe in this manner how in regions of space of spin density, Figure 3 (IIId).

The $\pi$ lengthC3-C2-C1 are the difference HOMO and LUMO in water for $\mathrm{PBE} / 6-31++\mathrm{G}(1)$, HOMO PBE/LANL2DZ(1) neutral form and cation, in Figure 2 (IId) and Figure 2 (IIId), HOMO PBE/SDD (1) in Figure 2 (IId) is characteristic for neutral form. 
The difference in the shape of the spin density within purine and pyrimidine (uracile) are in the fact the anion are describe in only figure and for LANL2DZ and SDD the active centre are the same.

$\mathrm{N} 7$ are the nucleophile site for adenine with $-0.803,-0.541$, -0.899 for NPA, Mulliken and CHELPG charge in gas phase; $-0.806,-0.618,-0.917$ in solvent water when are optimized with LSDA/6-31++G; N1 and N10, in the same figure (Ia andIb), are the second and third sites in gas phase and N13 and N1 in solvent water, Figure 4 (Ic). N10 and N13 are the probable site obtained with Fukui Function ${ }^{31}$ and experimental data ${ }^{32-36}$.

En la literatura ${ }^{31}$ for the electrophilic site the active site N1, $\mathrm{C} 2$ and $\mathrm{C} 5$ are not the same with Figure 4 (Ia, Ib, Ic, IA, IIB and IIIC), C5 are the active site of radical obtained with Fukui Function $^{31}$ and in this work,Figure 4 (Ia,Ic, IA, IB and IC) in the solvent water.

With PBE/6-31++G level of theory the active centers are the same, but in solvent water are N13, N10 and N7. The LANL2DZ and SDD relativist set bases are not influence in the results,N7, N1, N10 when the difference of the SDD are (0.001, 0.001, 0.001), for Mulliken charge N7, N1, N11 (0.003, 0.006, 0.018), CHELPG N7, N13, N10 (0.001, 0, $0.002)$.

For cytosine in Figure 5 (Ia, Ib, Ic, IA, IB and IC)N3, O13, $\mathrm{N} 2$ and N10 are the active centers for the electrophilic agents when the positions of the atoms is different with the level of theory and solvent water the exceptions being when the CHELPG charge are used.N3, N10 and O13 are the active centers when the PBE/6-31++G level of theory are employed, but when solvent water are used the active centers are N3, N2 and O13, Figure 5 (IIA, IIB and IIC). The PBE/LANL2DZ or $\mathrm{PBE} / \mathrm{SDD}$ level of theory are not influencing for the charge value, Figure 5 (IIIA, IIIB andIIIC) comparing with the PBE/6-31++G level. The difference between LANL2DZ and SDD for NPA charge N3, N10, O13 are (0.001, 0.001, 0.001); for Mulliken charge N3, N10, C6 are $(0.003,0.002,-0.011)$; for CHELPG N3, N2, O13 are $(0,-0.001,0),(-0.001,-0.001$, $0)$ in gas phase; for NPA charge N3, O13, N2 are $(0.001$, $0.001,0.001)$, for Mulliken charge N3, O13, N10 (0.002, $0.003,0.001)$, CHELPG charge are N3, N2, O13 (-0.001, $0.002,0)$ in solvent water.O13, C6, N2 are the active centers reported in literature ${ }^{31}$ for $\mathrm{LSDA} / 6-31++\mathrm{G}$.

When increasing the basicity in order $\mathrm{T} \leq \mathrm{A}<\mathrm{C}<\mathrm{G}$ the active centers in gas phase are N13, N4, N7 and in solvent water N13, O16 and N4 Figure 6 (Ia and IA).

In Figure $6(\mathrm{Ib})$ and (IB) N4, N13, N7 in gas phase is different of the value obtained in solvent water N13, O16, N4. When are used CHELPG the active centers not change in solvent water, N13, N4, N6 in gas phase and N13, N6 and N4 in solvent water. Only when the water are present the $\mathrm{O} 16$ to put in common the electrons but the N13 strive with he. With $\mathrm{PBE} / 6-31++\mathrm{G}$ the active centers are close with the previously level of theory. For guanine the solvent water when are used LANL2DZ or SDD the active centers obtained with Mulliken (exception O16) and CHELPG are not change, Figure 6 (Ia, Ib, Ic, IA, IB and IC), but for the value obtained with CHELPG are the little difference $(0.017,0.011,-0.011)$ for LANL2DZ and SDD. The difference between LANL2DZ and SDD for NPA charge are N13, N4, N6 (0.001, 0.001, $0.001)$, for Mulliken charge are N13, N4, N7 (0.004, -0.004, $0)$, for CHELPG charge are N13, N6, N4 (0, -0.001, -0.002) in gas phase; for NPA charge are N13, O16, N6 $(0.001,0$, 0.001 )

In the uracile with LSDA/6-31++G and PBE/6-31++G are not neither difference between NPA, Mulliken in gas phase but in solvent water the O8, O7, N9 are the nucleophylic centers. When I optimized with PBE/LANL2DZ are the difference for the active centers $(0.001,0,0.001),(-0.004$, $0.002,0.003),(-0.003,-0.001,0)$ in gas phase and $(0.001$, $0.001,0.001),(-0.005,-0.002,0.002),(-0.001,-0.001,-0.002)$ in solvent water with PBE/SDD.The differences of the bond lengths are very little for all structure.

For the calculation of the IPv in this work I take into consideration the stabilization energy of the ejected hydrated electron, the experimental ground-state of the "quasi-free" electron in the liquid $\left(-1.3 \mathrm{eV}^{37 \mathrm{y}}\right)$ was added in the calculation of the IPv according to the equation:

$$
\mathrm{IPV}=\mathrm{E}_{\mathrm{n}}-\mathrm{E}_{\mathrm{t}}+\mathrm{V}
$$

where $E_{n}$ is the total energy of the neutral molecule, $E_{t}$ is the total energy of the radical cation in the condensed phase, and $\mathrm{V}$ is the hydrated electron stabilization energy as defined above.

The bulk solvent polarization effects on the IPv and Ev are of the DNA/RNA bases, are presented in Table 2.

It was found that isolated nucleobases can stabilize the surplus electron in a dipole-bound state. In Table 7 I listed the dipole moments of the DNA and RNA bases when cytosine and uracil which have been calculated using the LSDA/6-31++G level of theory are underestimated compared to the experimental value ${ }^{37}$.

Table 1. Electronic proprieties of the DNA and RNA bases in the gas phase.

\begin{tabular}{|c|c|c|c|c|c|c|c|c|c|c|}
\hline Proprieties & Ipv \% (eV & & & & & Eav \% (eV & & & & \\
\hline Method employed / basis set & $6-31++G^{a}$ & LANL2DZ $^{a}$ & SDD $^{\mathrm{a}}$ & $631++G^{b}$ & $\operatorname{Exp}^{\mathrm{c}}$ & $6-31++G^{a}$ & LANL2DZ $^{a}$ & SDD $^{\mathrm{a}}$ & $631++G^{b}$ & $\operatorname{Exp}^{d}$ \\
\hline Adenine & -2.61 & 9.95 & 11.73 & 16.00 & 8.44 & 88.9 & 51.85 & 51.8 & -29.6 & -0.54 \\
\hline Cytosine & -4.36 & 7.49 & -5.37 & 2.80 & 8.94 & 306.3 & 521.88 & 165.6 & -240.6 & -0.32 \\
\hline Guanine & -4.98 & -5.70 & -5.83 & 1.58 & 8.24 & -115.2 & 260.87 & 300.0 & -115.2 & -0.46 \\
\hline Uracil & 10.63 & 9.68 & -2.42 & 6.21 & 9.50 & 81.8 & 95.45 & 100.0 & -263.6 & -0.22 \\
\hline
\end{tabular}


Table 1. Continued

\begin{tabular}{|c|c|c|c|c|c|c|c|c|}
\hline \multirow{2}{*}{$\begin{array}{l}\text { Proprieties } \\
\text { Method employed/basis set }\end{array}$} & \multicolumn{4}{|c|}{ Hardness (eV) } & \multicolumn{4}{|c|}{ Electronegativity (eV) } \\
\hline & $6-31++G^{a}$ & LANL2DZ $^{a}$ & SDD $^{\mathrm{a}}$ & $631++G^{b}$ & $6-31++G^{a}$ & LANL2DZ & SDD $^{\mathbf{a}}$ & $631++G^{b}$ \\
\hline Adenine & 4.62 & 5.05 & 5.13 & 5.09 & 3.60 & 4.23 & 4.31 & 4.71 \\
\hline Cytosine & 4.92 & 5.80 & 4.66 & 4.37 & 3.62 & 3.81 & 3.80 & 4.82 \\
\hline Guanine & 3.88 & 4.71 & 4.80 & 4.15 & 3.95 & 3.05 & 2.96 & 4.22 \\
\hline Uracil & 5.46 & 5.43 & 4.85 & 4.86 & 5.06 & 4.99 & 4.42 & 5.22 \\
\hline
\end{tabular}

a - all of the calculations are optimizedwith the PBE level

${ }^{b}$-all of the calculations are optimized with the LSDA level

c - experimental data [14]

d - experimental data [12]

Table 2. Electronic proprieties of the DNA and RNA bases in the solvent ( $\varepsilon=78.39)$.

\begin{tabular}{|c|c|c|c|c|c|c|c|c|c|}
\hline Proprieties & Ipv (eV) & & & & & $\operatorname{Eav}(e V)$ & & & \\
\hline Método / Set de Bases & $6-31++G^{a}$ & LANL2DZ $^{a}$ & SDD $^{\mathbf{a}}$ & $6-31++G b$ & expet & $6-31++G^{a}$ & LANL2DZ $^{a}$ & SDD $^{a}$ & $6-31++G^{b}$ \\
\hline Adenine & 4.84 & 4.79 & 4.78 & 5.42 & 5.0 & 1.43 & 1.30 & 1.29 & 2.11 \\
\hline Cytosine & 5.13 & 5.04 & 5.04 & 6.16 & 5.5 & 1.44 & 1.26 & 1.26 & 2.20 \\
\hline Guanine & 4.49 & 4.41 & 4.40 & 5.03 & 4.8 & 1.19 & 0.97 & 0.96 & 1.91 \\
\hline Uracil & 5.50 & 5.41 & 5.40 & 8.40 & - & 1.75 & 1.57 & 1.57 & 2.28 \\
\hline
\end{tabular}

Table 2. Continued

\begin{tabular}{|c|c|c|c|c|c|c|c|c|}
\hline \multirow{2}{*}{$\begin{array}{l}\text { Proprieties } \\
\text { Método / Set de Bases }\end{array}$} & \multicolumn{4}{|c|}{ Hardness (eV) } & \multicolumn{4}{|c|}{ Electronegativity $(\mathrm{eV})$} \\
\hline & $6-31++G^{a}$ & LANL2DZ $^{a}$ & SDD $^{a}$ & $6-31++G^{b}$ & $6-31++G^{a}$ & LANL2DZ $^{a}$ & SDD $^{a}$ & $6-31++G^{b}$ \\
\hline Adenine & 1.71 & 1.74 & 1.74 & 1.66 & 3.13 & 3.04 & 5.26 & 3.77 \\
\hline Cytosine & 1.85 & 1.89 & 1.89 & 1.98 & 3.29 & 3.15 & 3.80 & 4.18 \\
\hline Guanine & 1.65 & 1.72 & 1.72 & 1.56 & 2.84 & 3.30 & 3.76 & 3.47 \\
\hline Uracil & 1.87 & 1.92 & 1.92 & 3.06 & 3.62 & 3.49 & 4.14 & 5.34 \\
\hline
\end{tabular}

${ }^{\text {a }}$ - all of the calculations are optimized with the PBE level

${ }^{b}$ - all of the calculations are optimized with the LSDA level

c-experimental data

Table 3. Harmonic frequencies $\left(\mathrm{cm}^{-1}\right)$ of the DNA and RNA are optimizedwith LSDA/6-31++G.

\begin{tabular}{|c|c|c|c|c|c|c|}
\hline Nr. & adenine & expt & cytosine & expt & guanine & uracil \\
\hline 1 & 165 & 162 & 138 & 197 & 137 & 172 \\
\hline 2 & 216 & 214 & 202 & 232 & 177 & 183 \\
\hline 3 & 265 & 242 & 349 & 260 & 200 & 376 \\
\hline 4 & 304 & 276 & 429 & & 314 & 421 \\
\hline 5 & 515 & 298 & 480 & 330 & 316 & 516 \\
\hline 6 & 526 & 503 & 518 & & 331 & 534 \\
\hline 7 & 562 & 514 & 543 & 537 & 361 & 554 \\
\hline 8 & 571 & 521 & 573 & 575 & 486 & 654 \\
\hline 9 & 589 & 528 & 601 & 614 & 523 & 706 \\
\hline 10 & 614 & 566 & 684 & 637 & 542 & 724 \\
\hline 11 & 649 & 610 & 721 & & 626 & 779 \\
\hline 12 & 668 & 655 & 772 & 781 & 628 & 784 \\
\hline 13 & 707 & 672 & 777 & 818 & 648 & 816 \\
\hline 14 & 724 & 678 & 808 & & 655 & 961 \\
\hline 15 & 818 & 802 & 926 & & 667 & 968 \\
\hline 16 & 843 & 848 & 954 & & 717 & 1086 \\
\hline 17 & 883 & 869 & 983 & & 734 & 1204 \\
\hline 18 & 937 & 927 & 1067 & 1090 & 793 & 1266 \\
\hline 19 & 940 & 958 & 1117 & & 810 & 1358 \\
\hline 20 & 996 & 1005 & 1221 & 1192 & 826 & 1384 \\
\hline 21 & 1084 & 1037 & 1273 & 1244 & 939 & 1415 \\
\hline 22 & 1146 & 1103 & 1349 & 1337 & 1016 & 1480 \\
\hline 23 & 1234 & 1181 & 1426 & 1422 & 1062 & 1650 \\
\hline
\end{tabular}




\begin{tabular}{lllllll}
\hline Nr. & adenine & expt & cytosine & expt & guanine & uracil \\
\hline 24 & 1251 & 1240 & 1523 & 1475 & 1079 & 1708 \\
25 & 1328 & 1268 & 1550 & 1539 & 1140 & 1736 \\
26 & 1358 & 1328 & 1613 & 1595 & 1166 & 3172 \\
27 & 1378 & 1340 & 1680 & 1656 & 1290 & 3215 \\
28 & 1398 & 1374 & 1714 & 1720 & 1330 & 3512 \\
29 & 1436 & 1419 & 3161 & & 1354 & 3556 \\
30 & 1467 & 1448 & 3183 & 3441 & 1387 & 1435 \\
31 & 1450 & 1482 & 3524 & & 1481 & \\
32 & 1591 & 1584 & 3533 & & 1543 & 1585 \\
33 & 1629 & 1606 & 3673 & & 1622 & 1656 \\
34 & 1684 & 1633 & & & 1747 \\
35 & 3164 & 3041 & & & 3240 \\
36 & 3239 & 3057 & & & 3492 \\
37 & 3523 & 3442 & & & 3550 \\
38 & 3586 & 3499 & & & 3583 \\
39 & 3666 & 3557 & & & 3687 \\
40 & & & & & \\
41 & & & & & & \\
42 & & & & & & \\
\hline
\end{tabular}

Table 4. Harmonic frequencies $\left(\mathrm{cm}^{-1}\right)$ of the DNA and RNA are optimized with $P B E / 6-31++G$.

\begin{tabular}{|c|c|c|c|c|c|c|}
\hline Nr. & adenine & expt & cytosine & expt & guanine & uracil \\
\hline 1 & 163 & 162 & 136 & 197 & 132 & 168 \\
\hline 2 & 214 & 214 & 198 & 232 & 166 & 177 \\
\hline 3 & 270 & 242 & 337 & 260 & 191 & 369 \\
\hline 4 & 297 & 276 & 413 & & 276 & 413 \\
\hline 5 & 508 & 298 & 427 & 330 & 305 & 511 \\
\hline 6 & 519 & 503 & 497 & & 317 & 525 \\
\hline 7 & 530 & 514 & 521 & 537 & 355 & 544 \\
\hline 8 & 556 & 521 & 553 & 575 & 471 & 637 \\
\hline 9 & 560 & 528 & 576 & 614 & 494 & 698 \\
\hline 10 & 603 & 566 & 665 & 637 & 501 & 709 \\
\hline 11 & 621 & 610 & 711 & & 599 & 756 \\
\hline 12 & 651 & 655 & 730 & 781 & 614 & 759 \\
\hline 13 & 682 & 672 & 751 & 818 & 632 & 804 \\
\hline 14 & 702 & 678 & 768 & & 640 & 945 \\
\hline 15 & 801 & 802 & 885 & & 661 & 953 \\
\hline 16 & 824 & 848 & 939 & & 690 & 975 \\
\hline 17 & 867 & 869 & 942 & & 708 & 1072 \\
\hline 18 & 921 & 927 & 1026 & 1090 & 768 & 1183 \\
\hline 19 & 931 & 958 & 1099 & & 792 & 1237 \\
\hline 20 & 983 & 1005 & 1203 & 1192 & 794 & 1346 \\
\hline 21 & 1067 & 1037 & 1215 & 1244 & 903 & 1378 \\
\hline 22 & 1114 & 1103 & 1331 & 1337 & 992 & 1391 \\
\hline 23 & 1212 & 1181 & 1392 & 1422 & 1018 & 1458 \\
\hline 24 & 1227 & 1240 & 1484 & 1475 & 1054 & 1611 \\
\hline 25 & 1285 & 1268 & 1501 & 1539 & 1087 & 1661 \\
\hline 26 & 1315 & 1328 & 1608 & 1595 & 1132 & 1684 \\
\hline 27 & 1342 & 1340 & 1643 & 1656 & 1266 & 3174 \\
\hline 28 & 1370 & 1374 & 1658 & 1720 & 1307 & 3217 \\
\hline 29 & 1398 & 1419 & 3160 & & 1318 & 3524 \\
\hline 30 & 1434 & 1448 & 3191 & & 1337 & 3567 \\
\hline 31 & 1463 & 1482 & 3539 & 3441 & 1385 & \\
\hline 32 & 1572 & 1584 & 3542 & & 1441 & \\
\hline 33 & 1599 & 1606 & 3700 & 3565 & 1489 & \\
\hline 34 & 1645 & 1633 & & & 1548 & \\
\hline 35 & 3172 & 3041 & & & 1583 & \\
\hline 36 & 3243 & 3057 & & & 1629 & \\
\hline 37 & 3534 & 3442 & & & 1690 & \\
\hline 38 & 3590 & 3499 & & & 3238 & \\
\hline 39 & 3676 & 3557 & & & 3506 & \\
\hline 40 & & & & & 3564 & \\
\hline 41 & & & & & 3592 & \\
\hline 42 & & & & & 3714 & \\
\hline
\end{tabular}


Table 5. Harmonic frequencies $\left(\mathrm{cm}^{-1}\right)$ of the DNA and RNA are optimized with PBE/LANL2DZ

\begin{tabular}{|c|c|c|c|c|c|c|}
\hline Nr. & adenine & expt & cytosine & expt & guanine & uracil \\
\hline 1 & 159 & 162 & 136 & 197 & 132 & 163 \\
\hline 2 & 209 & 214 & 198 & 232 & 166 & 175 \\
\hline 3 & 258 & 242 & 337 & 260 & 191 & 360 \\
\hline 4 & 295 & 276 & 413 & & 276 & 408 \\
\hline 5 & 498 & 298 & 427 & 330 & 305 & 497 \\
\hline 6 & 509 & 503 & 497 & & 317 & 515 \\
\hline 7 & 515 & 514 & 521 & 537 & 355 & 534 \\
\hline 8 & 546 & 521 & 553 & 575 & 471 & 629 \\
\hline 9 & 554 & 528 & 576 & 614 & 494 & 698 \\
\hline 10 & 594 & 566 & 666 & 637 & 501 & 708 \\
\hline 11 & 642 & 610 & 712 & & 599 & 744 \\
\hline 12 & 652 & 655 & 729 & 781 & 614 & 772 \\
\hline 13 & 672 & 672 & 751 & 818 & 632 & 807 \\
\hline 14 & 693 & 678 & 768 & & 640 & 933 \\
\hline 15 & 796 & 802 & 884 & & 661 & 948 \\
\hline 16 & 823 & 848 & 939 & & 690 & 960 \\
\hline 17 & 847 & 869 & 942 & & 708 & 1063 \\
\hline 18 & 903 & 927 & 1025 & 1090 & 768 & 1174 \\
\hline 19 & 932 & 958 & 1098 & & 792 & 1229 \\
\hline 20 & 968 & 1005 & 1202 & 1192 & 794 & 1335 \\
\hline 21 & 1064 & 1037 & 1215 & 1244 & 903 & 1367 \\
\hline 22 & 1105 & 1103 & 1330 & 1337 & 992 & 1379 \\
\hline 23 & 1201 & 1181 & 1393 & 1422 & 1018 & 1444 \\
\hline 24 & 1220 & 1240 & 1483 & 1475 & 1054 & 1608 \\
\hline 25 & 1289 & 1268 & 1501 & 1539 & 1087 & 1664 \\
\hline 26 & 1313 & 1328 & 1607 & 1595 & 1132 & 1681 \\
\hline 27 & 1333 & 1340 & 1642 & 1656 & 1266 & 3176 \\
\hline 28 & 1366 & 1374 & 1658 & 1720 & 1307 & 3220 \\
\hline 29 & 1390 & 1419 & 3160 & & 1318 & 3526 \\
\hline 30 & 1433 & 1448 & 3191 & & 1337 & 3567 \\
\hline 31 & 1456 & 1482 & 3539 & 3441 & 1385 & \\
\hline 32 & 1560 & 1584 & 3542 & & 1441 & \\
\hline 33 & 1581 & 1606 & 3699 & 3565 & 1489 & \\
\hline 34 & 1633 & 1633 & & & 1548 & \\
\hline 35 & 3166 & 3041 & & & 1583 & \\
\hline 36 & 3237 & 3057 & & & 1629 & \\
\hline 37 & 3541 & 3442 & & & 1690 & \\
\hline 38 & 3595 & 3499 & & & 3238 & \\
\hline 39 & 3696 & 3557 & & & 3506 & \\
\hline 40 & & & & & 3564 & \\
\hline 41 & & & & & 3592 & \\
\hline 42 & & & & & 3714 & \\
\hline
\end{tabular}

Table 6. Harmonic frequencies $\left(\mathrm{cm}^{-1}\right)$ of the DNA and RNA are optimized with PBE/SDD.

\begin{tabular}{|c|c|c|c|c|c|c|}
\hline Nr. & adenine & expt & cytosine & expt & guanine & uracil \\
\hline 1 & 159 & 162 & 136 & 197 & 132 & 163 \\
\hline 2 & 209 & 214 & 198 & 232 & 166 & 175 \\
\hline 3 & 257 & 242 & 337 & 260 & 191 & 360 \\
\hline 4 & 295 & 276 & 413 & & 274 & 407 \\
\hline 5 & 498 & 298 & 426 & 330 & 305 & 497 \\
\hline 6 & 509 & 503 & 497 & & 316 & 515 \\
\hline 7 & 513 & 514 & 521 & 537 & 355 & 533 \\
\hline 8 & 546 & 521 & 553 & 575 & 471 & 628 \\
\hline 9 & 553 & 528 & 575 & 614 & 493 & 697 \\
\hline 10 & 594 & 566 & 664 & 637 & 501 & 707 \\
\hline 11 & 642 & 610 & 710 & & 598 & 744 \\
\hline 12 & 651 & 655 & 729 & 781 & 614 & 772 \\
\hline 13 & 671 & 672 & 750 & 818 & 632 & 806 \\
\hline 14 & 693 & 678 & 767 & & 640 & 933 \\
\hline 15 & 796 & 802 & 884 & & 660 & 948 \\
\hline 16 & 823 & 848 & 939 & & 690 & 960 \\
\hline 17 & 846 & 869 & 942 & & 708 & 1062 \\
\hline 18 & 903 & 927 & 1026 & 1090 & 768 & 1174 \\
\hline 19 & 932 & 958 & 1098 & & 792 & 1229 \\
\hline 20 & 968 & 1005 & 1203 & 1192 & 794 & 1335 \\
\hline
\end{tabular}




\begin{tabular}{|c|c|c|c|c|c|c|}
\hline Nr. & adenine & expt & cytosine & expt & guanine & uracil \\
\hline 21 & 1064 & 1037 & 1215 & 1244 & 903 & 1367 \\
\hline 22 & 1105 & 1103 & 1331 & 1337 & 992 & 1379 \\
\hline 23 & 1201 & 1181 & 1392 & 1422 & 1018 & 1444 \\
\hline 24 & 1219 & 1240 & 1484 & 1475 & 1054 & 1608 \\
\hline 25 & 1288 & 1268 & 1501 & 1539 & 1086 & 1664 \\
\hline 26 & 1313 & 1328 & 1608 & 1595 & 1131 & 1683 \\
\hline 27 & 1333 & 1340 & 1642 & 1656 & 1265 & 3176 \\
\hline 28 & 1366 & 1374 & 1659 & 1720 & 1307 & 3221 \\
\hline 29 & 1390 & 1419 & 3161 & & 1318 & 3526 \\
\hline 30 & 1433 & 1448 & 3192 & & 1337 & 3567 \\
\hline 31 & 1456 & 1482 & 3538 & 3441 & 1385 & \\
\hline 32 & 1560 & 1584 & 3542 & & 1441 & \\
\hline 33 & 1581 & 1606 & 3699 & 3565 & 1490 & \\
\hline 34 & 1633 & 1633 & & & 1548 & \\
\hline 35 & 3167 & 3041 & & & 1583 & \\
\hline 36 & 3237 & 3057 & & & 1630 & \\
\hline 37 & 3541 & 3442 & & & 1691 & \\
\hline 38 & 3595 & 3499 & & & 3239 & \\
\hline 39 & 3696 & 3557 & & & 3505 & \\
\hline 40 & & & & & 3564 & \\
\hline 41 & & & & & 3591 & \\
\hline 42 & & & & & 3714 & \\
\hline
\end{tabular}

Table 7. Dipole moments (debey) of the DNA and RNA bases

\begin{tabular}{|c|c|c|c|c|}
\hline Level of theory/bases set & Adenine neutral/anion & Cytosine neutral/anion & Guanine neutral/anion & Uracil neutral/anion \\
\hline $\mathrm{LSDA} / 6-31++\mathrm{G}$ & $2.58(3.14)$ & $7.30(5.42)$ & $7.56(12.74)$ & $4.9(3.71)$ \\
\hline $\mathrm{PBE} / 6-31++\mathrm{G}$ & $2.52(3.46)$ & $7.20(5.77)$ & $7.38(4.44)$ & $4.84(5.18)$ \\
\hline PBE/LANL2DZ & $2.59(2.14)$ & $7.14(5.15)$ & $7.43(4.08)$ & $4.75(3.84)$ \\
\hline $\mathrm{PBE} / \mathrm{SDD}$ & $2.59(2.14)$ & $7.13(5.14)$ & $7.42(6.24)$ & $4.75(3.83)$ \\
\hline $\mathrm{LSDA} / 6-31++\mathrm{G}(\epsilon=78.39)$ & $3.79(3.74)$ & $11.3(8.81)$ & $11.43(14.70)$ & $7.13(6.42)$ \\
\hline $\mathrm{PBE} / 6-31++\mathrm{G}(\epsilon=78.39)$ & $3.72(3.62)$ & $11.03(8.76)$ & $11.23(14.56)$ & $7.07(6.45)$ \\
\hline PBE/LANL2DZ $(\epsilon=78.39)$ & $3.77(3.75)$ & $10.55(8.12)$ & $10.93(13.96)$ & $6.74(5.93)$ \\
\hline $\begin{array}{l}\mathrm{PBE} / \mathrm{SDD}(\epsilon=78.39) \\
\text { experimental data }^{\mathrm{x}}\end{array}$ & $3.77(3.76)$ & $10.54(8.11)$ & $10.93(13.96)$ & $6.73((5.93)$ \\
\hline
\end{tabular}

$\mathrm{x}$-in gase phase

\section{Conclusions}

N7, N10 and N4 are the most probable electrophylic center in adenine for the binding distances. The relativistic propertie not change the reactivity of the molecule. The cation structure of cytosine are very different of the neutral and anion structure. For adenine the spin density is positive for the electron of $\alpha$ spin when LSDA/6-31++G(G) and PBE/6$31++\mathrm{G}(\mathrm{g})$ are used. In the water the results are better. The spin orbitals are verry different of the HOMO and LUMO because are employed de 2 electron of the last orbital.

\section{References}

[1] Prashant Jain, Pradeep T., Biotecn. andBioing. 2005, 90, 1, pp. 59-63.

[2] Ivan Sondi, Branka S. Sondi, J. Colloidal Interface Science 2004, 275, pp. 177-182.

[3] Russo N., Toscamo M., Grand A., J. Am. Chem. Soc. 2001, 123, pp. 10272.

[4] Shuxi Dai, Xingtang Zhang, Tianfeng Li, et all., Applied Surface Science 2005, 249, 1-4, pp. 346-353.

[5] Famulari A., Moroni F., Sironi M., Gianinetti E., Raimondi M.,
J. Molec. Struct.(Theochem) 2000, pp. 529, 209.

[6] Ford G.P., Scribner J.D., Chem. Res. Toxicol.1990, 3, pp. 219.

[7] Cardona J.P., Lippard S.J., Gait M.J., Singh M., J. Am. Chem. Soc. 1982, 104, pp. 5793.

[8] Basch H., Krauss M., Stevens W.J., Cohen D., Inorg. Chem. 1986, 25, pp. 684.

[9] Nakayama N., Tanaka S., Kikuchi O., J. Theor. Biol. 2002, 215, pp. 13.

[10] J.R. Wiley, J.M.Robinson, S. Ehdaie, E.C.M. Chen, E.S.D. Chen, W.E. Wentworth, Biochem. Biophys.Res. Comm. 1991, 180 , pp. 841.

[11] E.C.M., Chen, E.S.D., W.E. Wentworth, Biochem. Biophys. Res. Commun.1990, 171, 97.

[12] K. Aflatooni, G.A. Gallup, P.D. Burrow, J. Phys. Chem. A. 1998, 102, pp. 6205.

[13] V.M. Orlov, A.N. Smirnov, Ya M. Varshavsky, Tetrahedron Lett. 1976, 17, pp. 4377-4378.

[14] N.S. Hush, S.C. Agnes, Chem. Phys. Lett. 1975, 34, pp. 11.

[15] D.S. Michael, B. Brent, C.A.-Odile, J. Phys. Chem. 1994, 99, pp. 1060.

[16] A.-O. Colson, B. Brent, M.D. Sevilla, J. Phys. Chem. 1993, 97, pp. 13852. 
[17] Carlos E. Crespo-Hernández, R. Arce, Y. Ishikawa, L. Gorb, J. Leszczynski, D.M. Close, J. Phys. Chem. A. 2004, 108, pp. 6373-6377

[18] A. Kumar, M.K. Mohammady, P.C. Mishra, S. Suhai, J. Comput. Chem. 2004, 25, pp. 1047.

[19] X Lee, Z. Cai, M.D. Sevilla, J. Phys. Chem A 2002, 106, pp. 9345.

[20] S.S. Wesolowski, M.L. Leininger, P.N. Pentchev, H.F. Schaefer, J. Am. Chem. Soc. 2001, 123, pp. 4023.

[21] S.D. Wetmore, R.J. Boyd, L.A. Enksson, Chem Phys. Lett. 2000, 322, pp. 129.

[22] M.D. Sevilla, B. Bosler, A.O. Colson, J. Phys. Chem. 1995, 99 pp. 1060.

[23] David M. Close, J. Phys. Chem. A. 2004, 108, pp. 1037610379.

[24] Ulf Norinder, J. Molecular Structure (Theochem) 1987, 151, pp. 259-269.

[25] Preuss M., Schmidt W.G., Seino K., Furthmuller J., Bechstedt F., J. Comput. Chem. 2004, 25, pp. 112.

[26] Fogarasi G., J. Phys. Chem A, 2002, 106, pp. 1381.

[27] Podolyan Y., Rubin Y.V., Leszczynski J., J. Phy.s Chem. A 2000, 104, pp. 9964.

[28] Peter Pulay,Svein S., Massimo M., Jon Baker,J. Comput. Chem. 2005, 26 (6), pp. 599-605.
[29] Xifeng Li, ZhongliCai, Michael D. Sevilla, J. Phys. Chem. A, 2002, 106, pp. 1596-1603.

[30] Attila Szabo, Neil S. Ostlund, "Modern Quantum Chemistry", Dover Publications, Inc., Mineola, New York, 1996, pp. 212

[31] M. Virginia Popa, Rev. Mex. Fís. 2007, 53(4), pp. 241-253.

[32] Irma N.Kolomietset.al., J. Molec. Struct., 1991, 250(1), pp. 111 .

[33] Hye-Young H. Kim, M. Cooper, LubomirV.N., Constance M.H. and Thomas M.H., Chem. Res. Toxicol. 2001, 14(9), pp. 1306-1314.

[34] Gareth K. Forde et al., J. Phys. Chem. A, 2006, 110(69), pp. 2308-2313.

[35] Marzili L.G., Kistenmacher and Eichhorn G.L., (Ed. John Wiley Sons, New York, 1980) pp.179.

[36] Fan J-Y Tercel M. and Denny W.A., Anti-Cancer Drug Design 1997, 12(4), pp. 277-293.

[37] J. Schiedt, R. Weinjauf, D.M. Neumark, E.W. Schlang, Chemical Physics 1998, pp. 511-524.

[38] Netmore S.D., Boyd R. J., Enksonn L.A., Chem. Phys. Lett., 322, 129, pp. 1997. 